\title{
Una experiencia en la enseñanza de la radicación con números enteros en un estudiante con limitación visual ${ }^{1}$
}

\author{
A teaching experience filing with integers \\ into a visually impaired student
}

\author{
A experiência de ensino de arquivamento com \\ números inteiros em um aluno com deficiência visual \\ Ángela María Moreno Muñoz ${ }^{2}$ \\ Ramiro Adolfo Jiménez Leal ${ }^{3}$
}

Recibido: mayo de 2013 Aceptado: agosto de 2013

\section{Resumen}

El presente documento da muestra de una forma de enseñar a estudiantes con limitación visual a obtener raíces de un número entero utilizando el ábaco de soroban, poniendo el caso específico de un estudiante. Teniendo en cuenta esto, el artículo se estructura en cinco partes, empezando por una contextualización, luego se hace uso de unos referentes teóricosprácticos básicos los cuales sustentan la forma de enseñanza, se prosigue con la descripción general de la experiencia obtenida con el estudiante, identificando así los logros y dificultades evidenciadas, finalmente se realiza una reflexión enfocada desde la importancia de plantear estrategias de enseñanza para un aula inclusiva.

Palabras clave: Estrategias de enseñanza; inclusión; alumno; necesidades especiales; alumnos discapacitados; limitación visual; matemáticas escolares; números; operaciones aritméticas; radicación; estructuras numéricas; números enteros; agrupación.

\begin{abstract}
This document is demonstrating a way to teach students with visual impairment to get roots of an integer using soroban abacus, putting the specific case of a student. Considering this, the article is divided into five parts, beginning with a contextualization, then makes use of some basic theoretical and practical references which support the form of teaching, continues with the overview of the experience with the student thus identifying the achievements and difficulties highlighted finally made a reflection focused from the importance of teaching strategies to raise an inclusive classroom.
\end{abstract}

Keywords: Teaching strategies, inclusion, student, special needs, students with disabilities, visual impairment, school mathematics, numbers, arithmetic operations, filing, numerical structures; integers; grouping.

1 Artículo de Investigación.

2 Universidad Distrital Francisco José de Caldas. Bogotá, Colombia. Contacto: morenangela_08@hotmail.com

1 Universidad Distrital Francisco José de Caldas. Bogotá, Colombia. Contacto: ramir3399@hotmail.com 


\section{Resumo}

Este documento está demonstrando uma forma de ensinar os alunos com deficiência visual para obter raízes de um inteiro usando soroban ábaco, colocando o caso específico de um aluno. Diante disso, o artigo está dividido em cinco partes, começando com uma contextualização, em seguida, faz uso de alguns referenciais teóricos e práticos básicos que sustentam a forma de ensino, continua com a visão da experiência com o aluno identificando, assim, as conquistas e as dificuldades destacadas finalmente fez uma reflexão focada a partir da importância de estratégias de ensino para levantar uma sala de aula inclusiva.

Palavras-chave: estratégias de ensino, a inclusão, estudante, necessidades especiais, estudantes com deficiência, deficiência visual, a matemática da escola, números, operações aritméticas, arquivamento, estruturas numéricas; inteiros; agrupamento.

\section{Contextualización}

Esta experiencia de la enseñanza de la radicación con números enteros es desarrollada con un estudiante de 16 años de edad con limitación visual del colegio José Félix Restrepo, del grado séptimo. Este estudiante es participe de un aula inclusiva en donde hay tanto personas invidentes como videntes.

Resaltando lo anterior se destaca la importancia de implementar estrategias en la enseñanza que funcionen para todo el grupo estudiantil que estén dadas desde presentar un material que puedan manejar hasta la forma de explicar.

En la actualidad no son muy vistas las estrategias educativas para un aula inclusiva. Por ello este documento muestra una manera de llevar a cabo el aprendizaje del concepto matemático de radicación con números enteros mediante el trabajo con un material concreto (el ábaco de soroban) que puede ser manejado por todos los estudiantes. Por otro lado, se desarrolla una descripción con el mismo material reemplazando así a una clase de forma expositiva, en donde se presentan momentos en los cuales no todos los estudiantes pueden abstraer las explicaciones.

\section{Referentes teórico-prácticos básicos}

Es claro que dentro de las matemáticas se pueden utilizar variados recursos para la comprensión de algunos conceptos. En este trabajo adherimos al planteamiento de Rosich, Núñez \& Fernández (1996) quienes señalan que un estudiante llega a la abstracción a partir de la manipulación de lo concreto, puesto que los conceptos se aprenden al ir descubriéndolos. Por lo tanto, si queremos hacer un diseño de enseñanza inclusiva con estudiantes videntes e invidentes, necesitamos utilizar un modelo universal que permita que todos los estudiantes de la clase puedan aprender sin establecer diferencias en cuanto al uso de los recursos ni al desarrollo de la clase.

Lo anterior no quiere decir que no se necesite de un guía o mediador entre el saber y el estudiante. El docente es quien media esa relación. Braslavsky (s.f.) partiendo de la teoría de Vigotsky (precursor de la pedagogía inclusiva), fundamenta la importancia del papel docente cuando indica la necesidad de utilizar la experiencia ajena como instrumento de enseñanza. Gómez (2002) por otra parte, señala que si bien el estudiante es quien construye sus propias estructuras cognitivas, el profesor colabora en esa construcción implementando nuevas metodologías que superen la enseñanza tradicional.

En este estudio, nos apoyamos en el ábaco de soroban como un recurso metodológico que permite descubrir conocimientos debido a que se pueden realizar diferentes operaciones matemáticas (sumas, restas, multiplicaciones, divisiones, etc.) a partir de unas cuentas (pepitas) que se corren y forman las cantidades numéricas en un sistema decimal, éstas se 
encuentran dividas por una barra horizontal localizándose de la siguiente manera: en la parte inferior de la barra cuentas del 1 al 4 y en la parte superior una cuenta que indica el número $5,50,500, \ldots$ según el valor posicional. Este ábaco es considerado como un instrumento de acción-reflexión atractivo para la actividad mental de los estudiantes que hace posible la comprensión de los procesos llevados con las operaciones matemáticas (Siguero \& Carrillo, 1996, p. 53). Las operaciones se realizan a partir del proceso de descomposición de números en factores primos para llegar a obtener el valor entero de una raíz, esto requiere manejar las operaciones básicas de suma, multiplicación y división. La división se muestra a través de la descomposición de un entero positivo en factores primos (teorema fundamental de la aritmética) y estos factores en el momento de realizar la agrupación se pueden expresar como potencias. Jiménez (2004) resalta que la operación inversa a la radicación es la potenciación, de esta manera se mantiene una equivalencia entre $V_{y} \_\wedge_{\text {_. }}$. Por ello, el proyecto busca que los estudiantes realicen agrupaciones de los factores primos de la cantidad subradical buscando así una equivalencia con la raíz para poder solucionar determinado ejercicio.

Tomando todo lo anterior el docente a partir de la interacción con el estudiante en la forma de utilizar el instrumento y de dar un paso a paso para llegar a adquirir la raíz de un valor entero positivo, se muestra como mediador entre el saber adquirido por medio del recurso y de esta manera llega a reflejar la importancia de su trabajo.

\section{Descripción general de la experiencia de aula}

Este trabajo fue aplicado a un estudiante con limitación visual que no había comprendido la forma de hallar el valor entero de una raíz, partiendo de una cantidad subradical positiva; en una clase inclusiva en donde el tema sobre radicación se explicó de manera expositiva haciendo uso del marcador y el tablero. Debido a esto se decide emplear otra estrategia utilizando el ábaco de soroban como recurso en un apoyo extra-escolar, de esta manera se procede a que el estudiante obtenga el valor de las raíces descomponiendo el número en factores primos.
Dentro de la ejecución de la descomposición el estudiante tomaba dos ábacos, en uno iba reduciendo el número y en el otro iba colocando los factores primos que iba obteniendo. A continuación se le explicaba al alumno que de acuerdo al índice de la raíz que le daban debía sacar las potencias con los factores primos hallados y de esta manera podría encontrar la respuesta. Para hallar las potencias se le decía por ejemplo: con la raíz cuadrada, cuántos grupos de dos se pueden formar con el factor dos, factor tres,...etc., (de acuerdo a los factores encontrados dado el subradical). Al encontrar el número de grupos con cada factor se procedía a que multiplicara el número de grupos encontrados por el mismo factor y luego se multiplicaran los resultados, de esta manera se llegaba a encontrar la raíz. Uno de los ejercicios planteados fue $\sqrt{ }$, el estudiante primero descompuso la cantidad subradical 144 en factores primos encontrando así los siguientes: 2, 2, 2, 2, 3 y 3 . Luego al pedirle que formara grupos de dos (grupos de dos porque ese era el índice del radical) con el factor 2 y el factor 3 , obtuvo dos grupos con el factor $2\left(\wedge_{-} \_\right.$y un grupo con el factor 3 (_^_), al multiplicar el número de grupos obtenidos de acuerdo al factor por el factor se obtuvo 2 (grupos) $\times 2($ factor $)=4$ y 1 (grupo) $\times 3$ (factor $)=3$ y finalmente multiplicó los resultados de los factores $4 \times 3=12$ y de esta forma llego a determinar que $\sqrt{ }$.

\section{Logros y dificultades evidenciadas}

El estudiante se vale del instrumento para realizar de forma eficiente el proceso llevado a cabo con la descomposición de un número en factores primos para obtener el valor entero de una raíz, sin importar el índice de la misma, pues de acuerdo a ella reconoce la agrupación que debe hacer con los factores primos resultantes de la descomposición. En cuanto a las dificultades se observó que en algunas oportunidades, el estudiante olvida llevar en cuenta y marcar en el otro ábaco el factor primo obtenido, lo que le impidió encontrar la raíz entera y causó la repetición del proceso una o varias veces más. En otros casos, tenía en cuenta el recurso y se apropiaba de él para llegar a obtener una solución pero durante la agrupación de los factores no llegaba a indicar la expresión como una potencia. 


\section{Reflexión final.}

Dentro de la educación es primordial tener en cuenta las necesidades de los estudiantes en el proceso de enseñanza aprendizaje, pues no solo presentan necesidades educativas aquellas personas que sufren algún tipo de discapacidad (físico, cognitiva). La realidad de un aula se ve invadida en muchas ocasiones por una densa nube de dudas o vacíos que no permiten que los alumnos comprendan los conceptos matemáticos y por ende en ocasiones no llegan a solucionar problemas o ejercicios que involucren el tema.

Con esto se refleja que la realidad en las aulas de clase se ve invadida por las necesidades que presentan todos los estudiantes. En consecuencia, debemos atender esta situación buscando enseñar a partir de estrategias que utilicen materiales concretos que marquen la diferencia con las clases expositivas. El ábaco resultó ser una herramienta atractiva para realizar operaciones, y se pueden generar reflexiones a partir de la manipulación.

\section{Referencias}

Braslavsky, B. (s.f.). El ciego en la teoría de Vigotsky. Discapacidad visual hoy. Recuperado de http:// www.juntadeandalucia.es/averroes/caidv/interedvisual/dvh_07/dvh_07_17.pdf

Gómez J. (2002). De la enseñanza al aprendizaje de las matemáticas. Barcelona: Paidós.

Jiménez, D. (2004). Álgebra. La magia del símbolo. Caracas: CEC, SA.

Rosich, N., Núñez, J. M. \& Fernández, J. E. (1996). Matemáticas y deficiencia sensorial. Madrid: síntesis.

Siguero, F. H. \& Carrillo, E. (1996). Recursos en el aula de matemáticas. Madrid: síntesis. 